\title{
Physiological, mitochondrial, and oxidative stress differences in the presence or absence of lactation in rats
}

\author{
Hayden W. Hyatt', Yufeng Zhang'², Wendy R. Hood² and Andreas N. Kavazis ${ }^{{ }^{*}}$
}

\begin{abstract}
Background: Human epidemiological data show that breastfeeding reduces the mother's probability of developing several disease conditions, including obesity and type II diabetes compared to mothers that give birth but do not breastfeed. The goal of this investigation was to characterize how lactation changes a rat's body composition, metabolism, mitochondrial function, and oxidative stress.

Methods: Ten-week old female Sprague-Dawley rats were divided into three groups ( $n=8$ per group): 1 ) non-reproductive $(N R), 2$ ) those that were allowed to mate and give birth, but were not allowed to suckle their pups (PP), and 3) those that were allowed to mate and give birth, and suckled their young until weaning at 21 days (PL). All animals were sacrificed at a time corresponding to 7 days following the weaning of pups (i.e., day 28 postpartum).

Results: The body mass of PL rats was similar to NR rats, but the body mass of PP rats was higher than NR rats. Importantly, PL rats had lower retroperitoneal white adipose tissue mass compared to both NR and PP rats. The difference in fat mass was accompanied by higher protein levels of PPARס, SOD2, and reduced oxidative damage. Furthermore, the liver of PL rats had higher mitochondrial function with NADH-linked substrates, and higher expression of PGC-1a, PPARS, and SOD2.

Conclusions: These acute differences observed between female rats that did and did not suckle their young could be used as the foundation for future research investigating the prolonged and sustained benefits of lactation.
\end{abstract}

Keywords: Lactation, Metabolism, Mitochondria, Oxidative stress

\section{Background}

Exercise, fasting, and calorie restriction are examples of metabolic perturbations that induce physiological adaptations capable of promoting a healthy phenotype that is protective against various health disparities [1-4]. However, investigators often overlook lactation as a challenging metabolic event. Indeed, epidemiological studies in humans suggest that breastfeeding benefits the mother by reducing the risk of obesity, type II diabetes, hypertension, and several types of cancer [5-9]. Yet, why these health benefits may accrue to breastfeeding are largely unknown.

During reproduction, a female's metabolism goes through two dramatic changes. With the onset of pregnancy, a female's metabolic processes are primed to support glucose

\footnotetext{
* Correspondence: ank0012@auburn.edu

'School of Kinesiology, Auburn University, 301 Wire Road, Auburn, Alabama 36849, USA

Full list of author information is available at the end of the article
}

and amino acid transport to the fetus. Glucose transport is facilitated by an increase in maternal insulin production and as a result, maternal cells often display increased resistance to insulin $[10,11]$. This change could also be associated with increases in circulating fatty acids and increased visceral adiposity [12]. In contrast, during lactation there is a decrease in insulin secretion, which is associated with a drop in $\beta$-cell proliferation, improved insulin sensitivity, and a shift in lipoprotein lipase and triacylglyceride levels that facilitate the mobilization of lipid precursors to be used for milk synthesis $[13,14]$. Based on current epidemiological data in humans and the limited physiological observations in animal models, Stuebe and Rich-Edwards proposed the reset hypothesis which posits that lactation plays a central role in mobilizing fat stores and resetting the risk of metabolic disease [9]. However, research evaluating the physiological differences between females that give birth and 
lactate versus those that give birth and do not participate in lactation are lacking.

White adipose tissue (WAT), liver, and skeletal muscle account for more than $50 \%$ of a non-reproductive adult's metabolic rate [15]. Thus, any physiological changes that regulate metabolism in these organs during lactation could contribute to potential protective effects conferred by lactation. In this regard, Gutgesell and collaborators showed that genes important in oxidative and lipid metabolism (i.e. peroxisome proliferator activated receptor alpha $[$ PPAR- $\alpha$, peroxisome proliferator activated receptor gamma coactivator 1 alpha $[\mathrm{PGC}-1 \alpha]$, and peroxisome proliferator activated receptor gamma coactivator 1 beta $[\mathrm{PGC}-1 \beta])$ displayed reduced mRNA expression in liver and skeletal muscle 2 weeks following the cessation of lactation when compared to animals that gave birth but did not suckle their young [16]. Additionally, Pichaud and collaborators reported decreased electron transport system activity in the liver during peak lactation in the mouse [17]. Mediation of oxidative metabolism and thus mitochondrial function also may have important health implications on states of oxidative stress. As such, the current study seeks to delineate the potential effects that lactation has on markers of metabolism, mitochondrial function, and oxidative stress. Specifically, we hypothesized that females that give birth and suckle their pups would have lower body mass due to enhanced lipid metabolism and lower blood glucose concentrations driven, in part, by enhanced mitochondrial signaling in liver and skeletal muscle. In addition, we hypothesized that the higher metabolic demand in lactation would not impose an increase in markers of oxidative damage, as suggested to be a consequence of reproduction [18].

\section{Methods}

\section{Animal husbandry}

All experimental procedures were approved by Auburn University's Institutional Animal Care and Use Committee (PRN 2014-2591). Ten-week old Sprague-Dawley rats were obtained from Envigo (Indianapolis, IN). Animals were acclimated with their diet and facility 10 days prior to the beginning of the experiment. Rats were housed under standard laboratory conditions $(46 \times 25 \times 20 \mathrm{~cm}$ boxes, $12 \mathrm{~L}: 12 \mathrm{D}$ cycle, $\left.22^{\circ} \mathrm{C}, 50 \% \mathrm{RH}\right)$, and given ad libitum access to food (Teklad Global Diet 2018, Envigo) and water. Female rats ( $n=8$ per group) were randomly assigned to one of three treatment groups: 1) nonreproductive (NR), 2) those that were allowed to mate and became pregnant, but were not allowed to suckle their pups (PP), and 3) those that were allowed to mate, became pregnant, and suckled their young for 21 days (PL). Female rats were paired in their boxes with same-group counterpart, but separated during late pregnancy and lactation to prevent cross-fostering of pups after birth. Pups were removed from females in the PP group within
$12 \mathrm{~h}$ of birth. Litter size was adjusted to eight on the day of parturition pups, and were weaned and removed from the box on day 21 for females in the PL group. All animals were sacrificed at a time corresponding to 7 days following the weaning of pups (i.e., day 28 postpartum), allowing PL females to return to a non-reproductive state.

\section{Blood collection and analysis}

Rats were fasted for $4 \mathrm{~h}$ and then were anesthetized using isoflurane vapors and body mass was quickly recorded. The anesthetized animals were than decapitated, and blood was collected, allowed to clot, and then centrifuged. Following centrifugation the serum was frozen at $-80{ }^{\circ} \mathrm{C}$ for subsequent analyses. Serum glucose (STA-680, Cell Biolabs, San Diego, CA, USA) and non-esterified fatty acids (NEFA) (STA-618, Cell Biolabs) were quantified using the manufacturer's specifications.

\section{Tissue collection and handling}

After the decapitation, the following tissues were excised and weighed: liver, triceps surae (calf muscle), and the retroperitoneal white adipose tissue (WAT). After the mass of each tissue was recorded, a sample of tissue from calf skeletal muscle and liver was used for mitochondrial isolation and the remainder of tissues were frozen in liquid nitrogen and stored at $-80{ }^{\circ} \mathrm{C}$ for subsequent analyses. Upon gross evaluation, mammary tissue was not observed for females in any of the groups suggesting that the regression of mammary tissue was nearly or fully complete for females in the PP and PL groups at the time of tissue collection.

\section{Mitochondrial isolation}

Mitochondrial isolations for muscle were performed as previously described [19]. Excised muscles ( $750 \mathrm{mg}$ ) were trimmed to remove fat and connective tissues, weighed, and placed in 10 volumes of solution I $(100 \mathrm{mM} \mathrm{KCl}$, $40 \mathrm{mM}$ Tris $\mathrm{HCl}, 10 \mathrm{mM}$ Tris base, $1 \mathrm{mM} \mathrm{MgSO}$, $0.1 \mathrm{mM}$ EDTA, $0.2 \mathrm{mM}$ ATP, and $2 \%$ (wt/vol) free fatty acid bovine serum albumin (BSA), $\mathrm{pH}$ 7.40). Muscles were minced with scissors and the mince was homogenized for $15 \mathrm{~s}$ with a polytron. Protease (Trypsin) was added $(5 \mathrm{mg} / \mathrm{g}$ wet muscle), and the digested mince was mixed continually for $7 \mathrm{~min}$. Digestion was terminated by the addition of an equal volume of solution I. The homogenate was centrifuged at $500 \mathrm{~g}$ for $10 \mathrm{~min}$ at $4{ }^{\circ} \mathrm{C}$ and the supernatant was rapidly decanted through a double layer of cheesecloth and centrifuged at $3500 \mathrm{~g}$ for $10 \mathrm{~min}$ at $4{ }^{\circ} \mathrm{C}$. The supernatant was discarded and the mitochondrial pellet was resuspended in solution I. The suspension was centrifuged at $3500 \mathrm{~g}$ for $10 \mathrm{~min}$ at $4{ }^{\circ} \mathrm{C}$. The supernatant was again discarded, and the pellet was resuspended in 10 volumes of solution II (similar to solution I, but without BSA). This resuspended pellet was subsequently centrifuged at $3500 \mathrm{~g}$ 
for $10 \mathrm{~min}$ at $4{ }^{\circ} \mathrm{C}$. The final pellet containing mitochondria was suspended in $250 \mu \mathrm{l}$ of a solution containing $220 \mathrm{mM}$ mannitol, $70 \mathrm{mM}$ sucrose, $10 \mathrm{mM}$ Tris $\mathrm{HCl}$, and $1 \mathrm{mM}$ EGTA, pH 7.40. Mitochondria from liver were isolated as previously described [20]. Briefly, liver $(\sim 750 \mathrm{mg})$ was weighed and placed in 10 volumes of solution III $(250 \mathrm{mM}$ sucrose, $5 \mathrm{mM}$ HEPES, and $1 \mathrm{mM}$ EGTA), minced with scissors and the mince was homogenized with a PotterElvehjem PTFE pestle and glass tube (2 passes). The homogenate was centrifuged at $500 \mathrm{~g}$ for $10 \mathrm{~min}$ at $4{ }^{\circ} \mathrm{C}$ and the supernatant was rapidly decanted through a double layer of cheesecloth and centrifuged at $3500 \mathrm{~g}$ for $10 \mathrm{~min}$ at $4{ }^{\circ} \mathrm{C}$. The supernatant was discarded and the mitochondrial pellet was resuspended in solution III. The suspension was centrifuged at $3500 \mathrm{~g}$ for $10 \mathrm{~min}$ at $4{ }^{\circ} \mathrm{C}$. The final pellet containing mitochondria was suspended in $250 \mu \mathrm{l}$ of a solution containing (in mM) 220 mannitol, 70 sucrose, 10 Tris $\mathrm{HCl}$, and 1 EGTA, $\mathrm{pH}$ 7.40. All procedures we performed on ice and all solutions were kept on ice $\left(4{ }^{\circ} \mathrm{C}\right)$.

\section{Isolated mitochondrial oxidative phosphorylation}

Mitochondrial oxygen consumption was measured as described by Messer et al. [21]. Briefly, mitochondrial oxygen consumption was measured polarographically in a respiration chamber (Hansatech Instruments, United Kingdom). Isolated mitochondria $(20 \mu \mathrm{L})$ were incubated with $1 \mathrm{ml}$ of respiration buffer adapted from Wanders et al. [22] (100 mM KCL, $50 \mathrm{mM}$ MOPS, $10 \mathrm{mM}$ $\mathrm{KH}_{2} \mathrm{PO}_{4}, 20 \mathrm{mM}$ glucose, $10 \mathrm{mM} \mathrm{MgCl} 2,1 \mathrm{mM}$ EGTA, and $0.2 \%$ fatty acid free $\mathrm{BSA} ; \mathrm{pH}=7.0$ ) at $37{ }^{\circ} \mathrm{C}$ in a respiratory chamber with continuous stirring. For state 3 respiration, $2 \mathrm{mM}$ pyruvate and $2 \mathrm{mM}$ malate (complex I substrates) or $5 \mathrm{mM}$ succinate (complex II substrate) was used in the presence of $0.25 \mathrm{mM} \mathrm{ADP}$, and state 4 respiration was recorded following the phosphorylation of ADP as described by Estabrook [23]. Respiratory control ratio (RCR) was calculated as state $3 /$ state 4 oxygen consumption. Respiration values were expressed as a ratio to citrate synthase to compensate for mitochondrial enrichment in the samples.

\section{Mitochondrial oxidant emission}

Oxidant emission by mitochondria was determined using the oxidation of the fluorogenic indicator Amplex Red (Molecular Probes, Eugene, OR) in the presence of horseradish peroxidase [24]. The assay was performed at $37{ }^{\circ} \mathrm{C}$ in 96 -well plates using succinate as the substrate. Specifically, this assay was developed based on the concept that horseradish peroxidase catalyzes the hydrogen peroxide-dependent oxidation of nonfluorescent Amplex Red to fluorescent Resorufin Red. Resorufin Red formation was monitored at an excitation wavelength of $545 \mathrm{~nm}$ and an emission wavelength of $590 \mathrm{~nm}$ using a multiwell plate reader fluorometer (Synergy H1, BioTek,
Winooski, VT, USA). We recorded the level of Resorufin Red formation, and hydrogen peroxide production was calculated with a standard curve.

\section{Enzymatic assays for electron transport chain complex activity}

Complex I (NADH dehydrogenase) enzyme activity (EC 1.6.5.3) was measured as a function of the decrease in absorbance from NADH oxidation by decylubiquinone before and after rotenone addition [25]. Complex II (succinate dehydrogenase) activity (EC 1.3.5.1) was measured as a function of the decrease in absorbance from 2,6-dichloroindophenol reduction [25]. Complex III (ubiquinol cytochrome $c$ oxidoreductase) activity (EC 1.10.2.2) was determined as a function of the increase in absorbance from cytochrome $c$ reduction [25]. Complex IV (cytochrome $c$ oxidoreductase) activity was determined as a function of the decrease in absorbance from cytochrome $c$ oxidation [25]. Specificity of complex IV activity was determined by monitoring changes in absorbance in the presence of $\mathrm{KCN}$ [25]. Citrate synthase (EC 4.1.3.7) was measured as a function of the increase in absorbance from 5,5'-dithiobis-2-nitrobenzoic acid reduction [25]. Enzyme activities were expressed as a ratio to citrate synthase to compensate for mitochondrial enrichment in the cell samples. All measurements were performed using the BioTek Synergy H1 spectrophotometer (BioTek, Winooski, VT, USA).

\section{Protein abundance}

The relative concentration of proteins was quantified by Western blot analysis [24]. To accomplish this, tissue was homogenized 1:10 (wt/vol) in $5 \mathrm{mM}$ Tris $\mathrm{HCl}$ ( $\mathrm{pH} 7.5)$ and 5 mM EDTA (pH 8.0), and protease inhibitor cocktail (14224-396, VWR, Radnor, PA, USA) and was centrifuged at $1500 \mathrm{~g}$ for $10 \mathrm{~min}$ at $4{ }^{\circ} \mathrm{C}$. Protein content of the supernatant was quantified by the method of Bradford [26]. Proteins were separated by polyacrylamide gel electrophoresis via 4-20\% polyacrylamide gels (BioRad, Hercules, CA, USA). After electrophoresis, the proteins were transferred to PVDF membranes. Nonspecific sites were blocked in phosphate-buffered saline (PBS) solution containing $0.1 \%$ Tween 20 and 5\% nonfat milk. Membranes were then incubated overnight at $4{ }^{\circ} \mathrm{C}$ with primary antibodies purchased from GeneTex (Irvine, CA, USA) directed against peroxisome proliferator activated receptor alpha (PPAR $\alpha$, GTX101096, 1:1000), peroxisome proliferator activated receptor delta (PPAR $\delta$, GTX113250, 1:2000), peroxisome proliferator activated receptor gamma, coactivator 1 alpha (PGC-1 $\alpha$, GTX37356, 1:1000), superoxide dismutase 1 (SOD1, GTX100554 1:2000), superoxide dismutase 2 (SOD2, GTX116093, 1:2000), catalase (CAT, GTX110704, 1:2000), and glutathione peroxidase (GPX, GTX116040, 1:2000). 
Following incubation with primary antibodies, membranes were washed with PBS-Tween $(5 \mathrm{~min} \times 3)$ and then incubated with secondary antibodies for $1 \mathrm{~h}$ in room temperature. After washing ( $5 \mathrm{~min} \times 3)$, a chemiluminescent system was used to detect labeled proteins (GE Healthcare, Buckinghamshire, UK). Images of the membranes were captured and analyzed by using the ChemiDoc-It2 Imaging System (UVP, LLC, Upland, CA). Protein expression was normalized to Ponceau staining.

\section{Assessment of indices of oxidative damage}

To determine the relative amount oxidative damage, we measured protein oxidation and lipid peroxidation. Lipid peroxidation was assessed by determining 4-hydroxynoneal (4-HNE; trans-4-hydroxy-2-nonenal, $\mathrm{C}_{9} \mathrm{H}_{16} \mathrm{O}_{2}$ ) expression via western blotting, as described above. Primary antibody for 4-HNE was purchased from Abcam (ab46545; 1:1000 dilution, Cambridge, MA, USA). Protein oxidation was measured by comparing relative expression of protein carbonyls using a commercially available kit (Oxy-Blot protein oxidation detection kit; Intergen, Purchase, NY, USA) via western blotting, as described by the manufacturer's instructions.

\section{Statistics}

Comparison between groups for each dependent variable were made by a one-way analysis of variance (ANOVA), with a Tukey post hoc test being used to determine significance differences between groups. However, in the case of state 3 respiration with succinate in muscle and serum FFA, the Brown-Forsythe test was significant and thus the Kruskal-Wallis test was performed, followed by the Dunn's post-hoc to determine significance differences between groups. Data are presented as mean $\pm \mathrm{SD}$, and significance was established at $p<0.05$.

\section{Results}

\section{Body and tissue mass}

During pregnancy and lactation, the body of females undergoes a large fluctuation in mass to accommodate the developing fetuses and active mammary tissue. The regression of these tissues 28 days following pregnancy and lactation varied between groups. Body mass was higher in PP compared to NR rats $(p=0.005)$ and the body mass of PP rats was higher than the mass of the PL rats, but this was not statistically different $(p=0.145)$ (Fig. 1a). Liver mass was higher in PL than NR rats $(p=0.004)$ (Fig. 1b). PL rats had lower retroperitoneal fat mass compared to both PP $(p=0.041)$ and NR rats $(p<0.001)$ (Fig. 1c). The combined mass of the rear triceps surae calf muscle was higher in PP than in NR rats $(p=0.027)$ (Fig. 1d).

\section{Serum glucose and NEFA concentrations}

The high energetic demand of lactation requires energy substrates to be delivered to metabolically active tissues via the blood stream. Two important metabolites transported in blood are glucose and NEFAs. Serum glucose concentration was lower in PL compared to NR $(p=0.029)$, but no statistical differences were detected in serum NEFA concentrations $(p=0.802)$ (Fig. 1e and $\mathrm{f}$ ).

\section{Mitochondrial respiration and oxidant emission}

RCR is a useful tool in assessing mitochondrial function, and can be used to measure respiration capacity of the mitochondria with different available substrates. Measuring RCR using isolated mitochondria provides the ability to observe ATP phosphorylation via electron shuttling at complex I and complex II. High-resolution respirometry was used in isolated mitochondria from liver and skeletal muscle. The RCR of liver mitochondria was higher in PL compared to PP and NR rats $(p=0.029$ and $p=0.002$, respectively) when using pyruvate and malate as the substrate (Fig. 2c). No statistical differences were detected in liver RCR between groups when succinate was used as the substrate ( $p=0.234$; Fig. $2 \mathrm{f})$. In addition, no statistical differences were detected in skeletal muscle RCR between groups when pyruvate and malate or succinate was used as the substrate ( $p=0.792$ and $p=0.996$, respectively) (Fig. $2 \mathrm{i}$ and l). Oxidant emission occurs during oxidative phosphorylation in the mitochondria, primarily at complex I and complex III sites [27]. No statistically differences $(p=0.191)$ were detected in oxidant emission from mitochondria isolated from liver (Fig. 2m). Oxidant emission from mitochondria isolated from skeletal muscle was lower in PP and PL compared to NR $(p=0.017$ and $p=0.008$, respectively) (Fig. 2n).

\section{Mitochondrial complex activity}

Phosphorylating ADP in the mitochondria is accomplished through electron pumps that harness the energy of electrons by oxidizing the reduced form of either NADH or FADH. Specifically, complex I transfers electrons from NADH to coenzyme Q10. Complex II transfers electrons from FADH. Complex III passes electrons down the chain by reducing cytochrome c. And, complex IV converts molecular oxygen into water and further shuttles protons into the intermembrane space. Activity of complex I in liver mitochondria was higher for both PP and PL rats compared to NR rats $(p=0.027$ and $p=0.046$, respectively) (Fig. 3a). Conversely, enzymatic activity of complex II in liver mitochondria was lower in PL rats than both NR and PP rats $(p=0.004$ and $p=0.0029$, respectively) (Fig. 3b). Complex III activity in liver mitochondria of PL rats was lower, albeit not statistically different, than PP and NR rats $(p=0.065$ and $p=0.075$, respectively) (Fig. $3 \mathrm{~b}$ ). Complex IV activity 

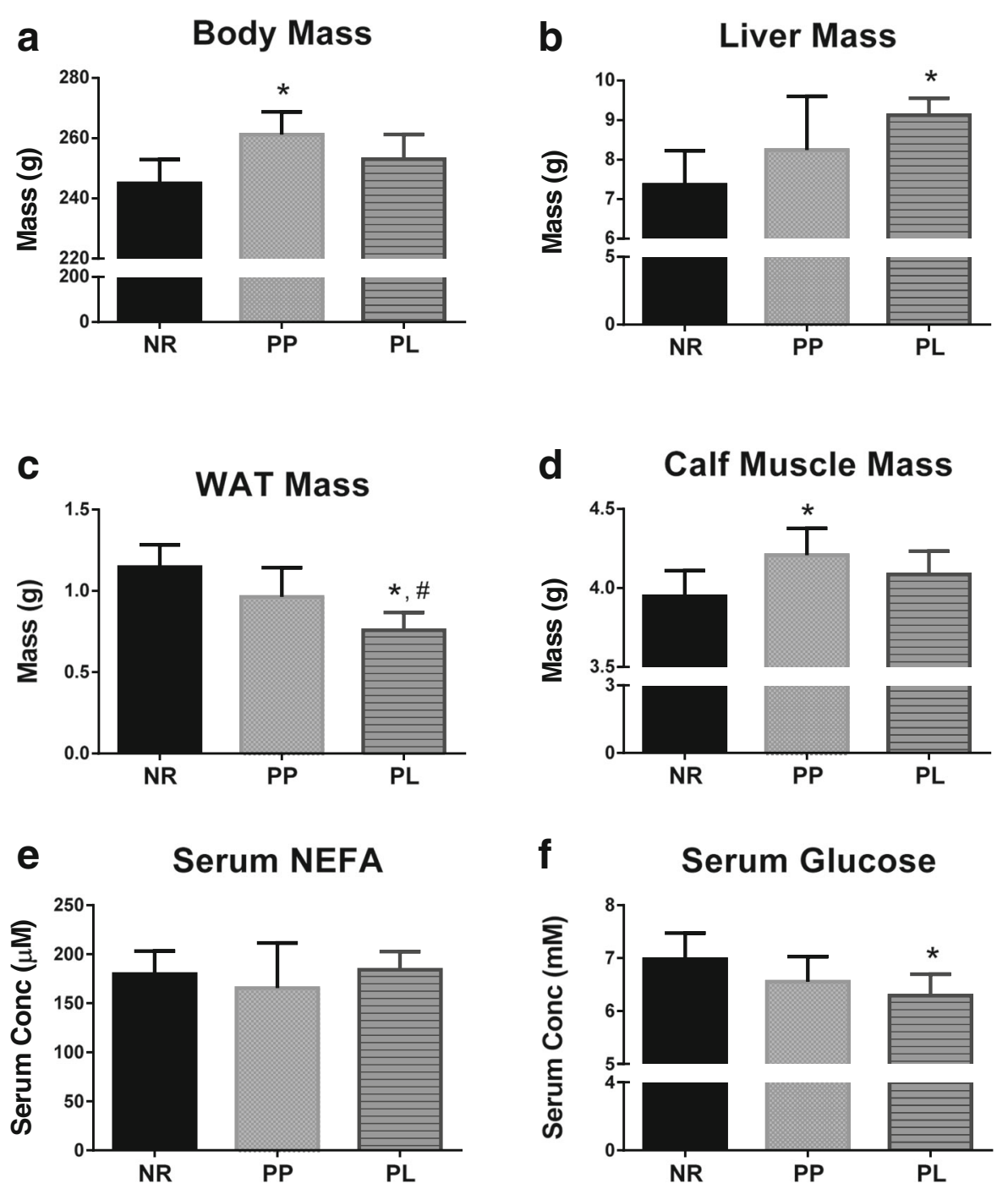

Fig. 1 Body mass, tissue mass, and serum metabolites for age-matched rats that did not reproduce (NR), and rats that did not (PP) or did (PL) suckle their young for 21 days postpartum. a Body mass, (b) liver mass, (c) retroperitoneal white adipose tissue (WAT) mass, (d) mass of both rear triceps surae (calf muscle mass), (e) serum concentration of non-esterified fatty acids (NEFA), and (f) serum concentration of glucose. Data shown are mean \pm SD. ${ }^{*}$ indicates different from NR $(p<0.05)$, and \# indicates different from PP $(p<0.05)$

was lower in liver mitochondria of PL compared to PP rats $(p=0.025$; Fig. 3d). Enzymatic activity of complex I and II were lower in mitochondria isolated from skeletal muscle in PL compared to NR rats $(p=0.044$ and $p=0.032$, respectively). (Fig. $3 \mathrm{e}$ and $\mathrm{f}$ ).

\section{Markers of oxidative and lipid metabolism}

The PPAR superfamily is associated with the regulation of genes involved in oxidative metabolism. Specifically PGC$1 \alpha$ is associated with regulation of genes involved in mitochondrial biogenesis [28] and PPAR $\delta$ is associated with genes involved in lipid and glucose metabolism [29, 30]. PGC- $1 \alpha$ liver protein expression was higher in PL rats compared to PP and NR rats $(p=0.035$ and $p=0.001$, respectively) (Fig. 4a). Also, PPAR $\delta$ liver protein expression was higher in PL compared to NR rats $(p=0.009)$ (Fig. 4b). No statistically differences were detected for PGC- $1 \alpha$ and PPAR $\delta$ in skeletal muscle $(p=0.331$ and $p=0.691$, respectively) (Fig. $4 \mathrm{c}$ and d). PPAR $\delta$ protein expression in WAT was higher in PL compared to PP and NR rats $(p<0.001)$ (Fig. 4e).

\section{Markers of oxidative stress}

The balance between oxidants and antioxidants is often referred to as oxidative stress. Endogenous antioxidant defense mechanisms exist to protect and detoxify oxidants. Specifically, SOD2 and SOD1 act to detoxify superoxide into the lesser harmful hydrogen peroxide. Hydrogen peroxide can be then be further detoxified by CAT and GPX. The protein levels of the antioxidants 

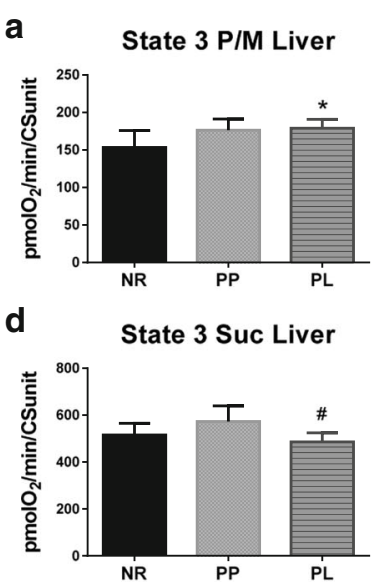

\section{g}

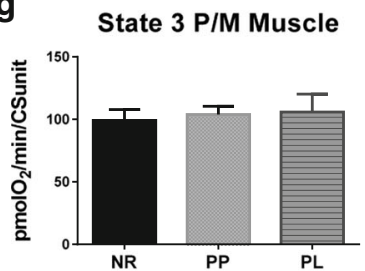

j

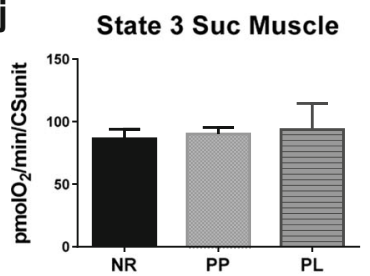

b

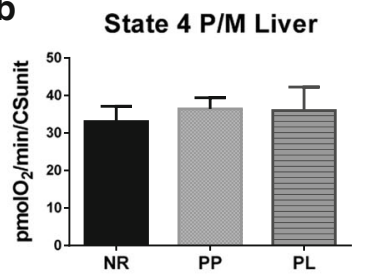

e

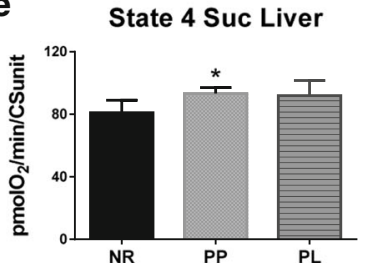

h

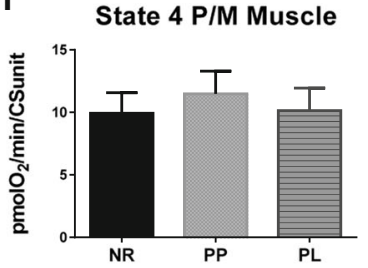

k

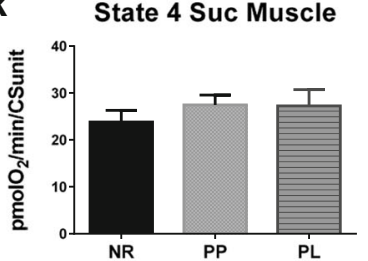

C

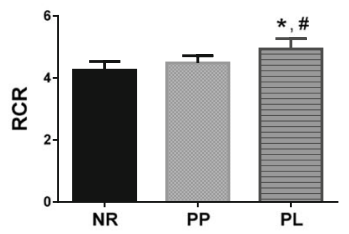

f

RCR Suc Liver

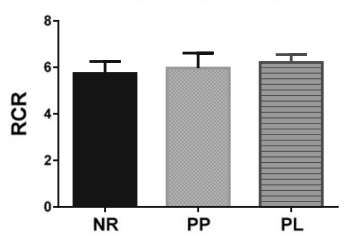

i

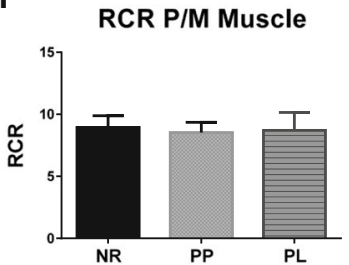

I RCR Suc Muscle

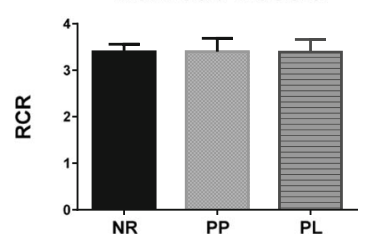

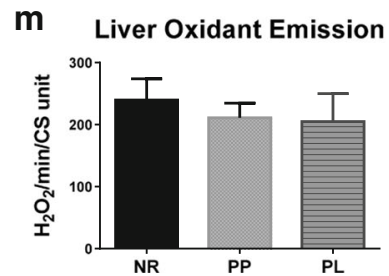

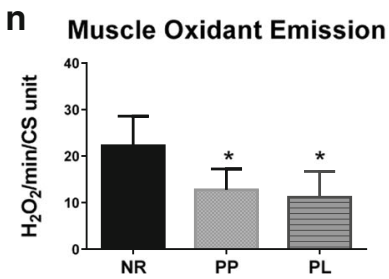

Fig. 2 Respiration and oxidant emission from mitochondria isolated from the liver and skeletal muscle of age-matched rats that did not reproduce (NR), and rats that did not (PP) or did (PL) suckle their young for 21 days postpartum. Data include liver (a) state 3 respiration utilizing complex I substrates (pyruvate and malate; $P / M$ ), (b) state 4 respiration utilizing $P / M$, (c) respiratory control ratio (RCR) utilizing $P$ / M), (d) state 3 respiration utilizing complex II substrates (succinate; suc), (e) state 4 respiration utilizing Suc, f RCR utilizing suc. Data also include skeletal muscle $(\mathbf{g})$ state 3 respiration utilizing P/M, (h) state 4 respiration utilizing P/M, (i) RCR utilizing P/M, (j) state 3 respiration utilizing suc, (k) state 4 respiration utilizing suc, (I) RCR utilizing suc. Finally (m) oxidant emission from liver and (n) oxidant emission from skeletal muscle are also presented. Oxygen consumption and hydrogen peroxide $\left(\mathrm{H}_{2} \mathrm{O}_{2}\right)$ rates were normalized to citrate synthase (CS). Data shown are mean \pm SD. ${ }^{*}$ indicates different from NR $(p<0.05)$, and \# indicates different from PP $(p<0.05)$

(SOD2, SOD1, CAT, and GPX) and markers of oxidative damage were compared between groups. Liver SOD2 protein levels were higher in PL compared to NR and PP rats ( $p=0.001$ and $p=0.025$, respectively) (Fig. 5a). Liver CAT protein expression was higher in PL and PP rats compared to NR rats $(p=0.015$ and $p=0.001$, respectively) (Fig. $5 c)$. No statistical differences $(p>0.05)$ were detected for SOD1, GPX, 4-HNE or protein carbonyls in liver (Fig. 5b and d-f). SOD2 protein levels were higher in WAT of PL compared to NR rats $(p=0.039)$ (Fig. 6a), and 4-HNE was lower in WAT of PL rats compared to NR rats $(p=0.004)$ (Fig. 6e). No statistical differences $(\mathrm{p}>0.05)$ were detected for SOD2, SOD1, CAT, GPX, 4-HNE, or protein carbonyls in skeletal muscle (Fig. 7). 
a

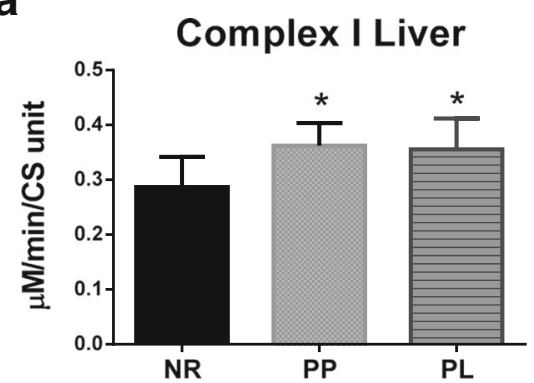

C

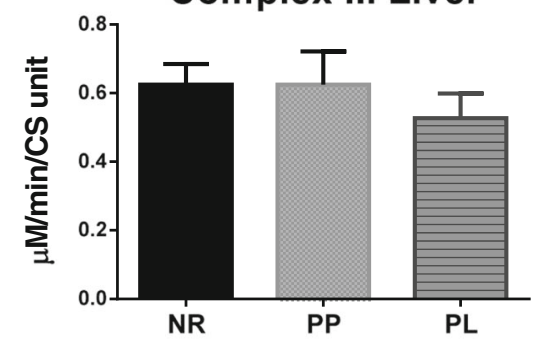

e

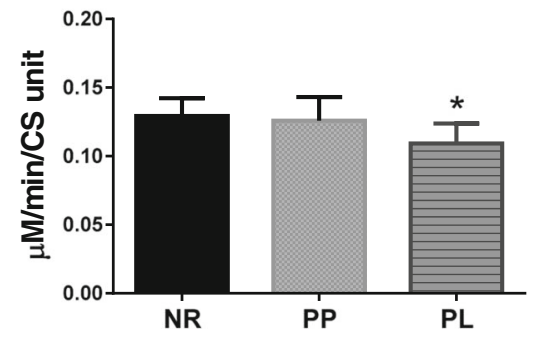

g
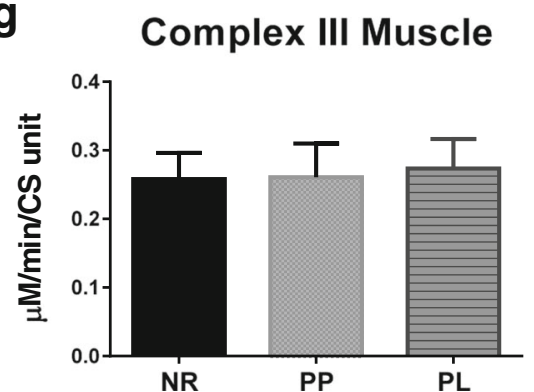

b

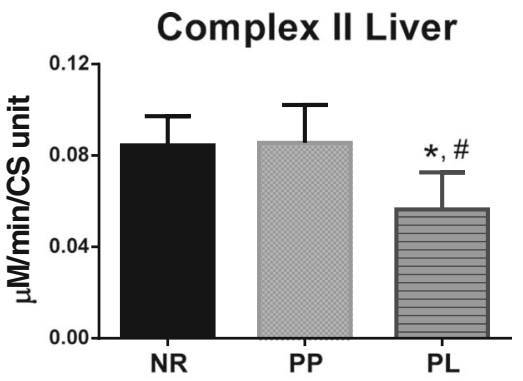

d

Complex IV Liver

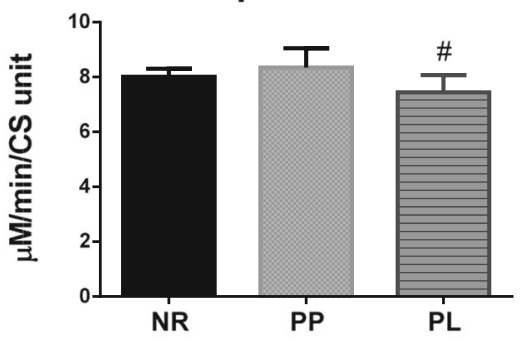

Complex II Muscle

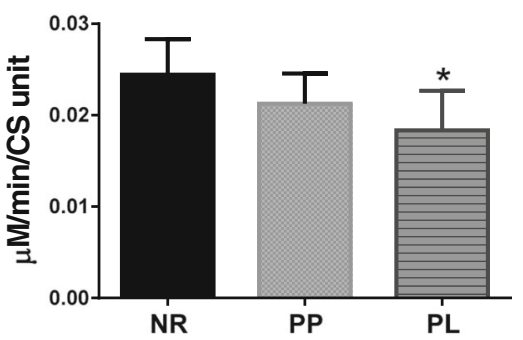

Complex IV Muscle

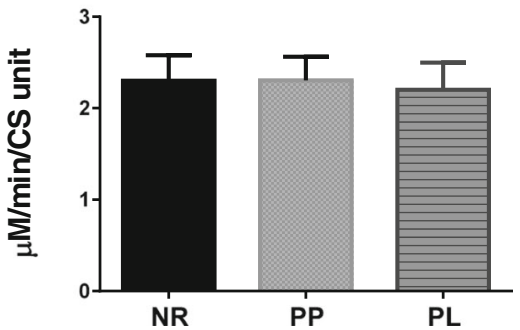

Fig. 3 Enzymatic activity of the mitochondrial complexes for liver and skeletal muscle of age-matched rats that did not reproduce (NR), and rats that did not (PP) or did (PL) suckle their young for 21 days postpartum. Data include (a) complex I, (b) complex II, (c) complex III, and (d) complex IV activity in the liver. In addition, data include (e) complex I, (f) complex II, (g) complex III, and (h) complex IV activity in skeletal muscle. Complex activity data are normalized to citrate synthase (CS). Data shown are mean \pm SD. * indicates different from NR $(p<0.05)$, and \# indicates different from PP $(p<0.05)$

\section{Discussion}

The metabolic changes that a female experiences during lactation are often the most dramatic physiological changes that she will experience in her lifetime. In this regard, many metabolic challenges (e.g., exercise, calorie restriction, etc.) have demonstrated to provide protective health benefits against diseases such as obesity and type II diabetes [1, 2, 31, 32]. Epidemiological data suggests that lactation contributes to a lower prevalence of obesity and type II diabetes, in addition to other health disparities [5-9]. Yet despite this knowledge, our understanding of the metabolic differences between females that maintain lactation and those that do not maintain lactation is limited. Our data suggest that metabolism and the bioenergetic capacity of females is enhanced following lactation in the rat. Specifically, we observed a reduced 


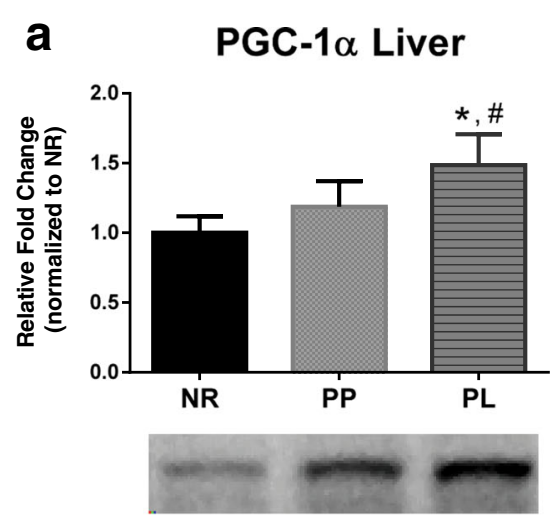

\section{b PPAR- $\delta$ Liver}
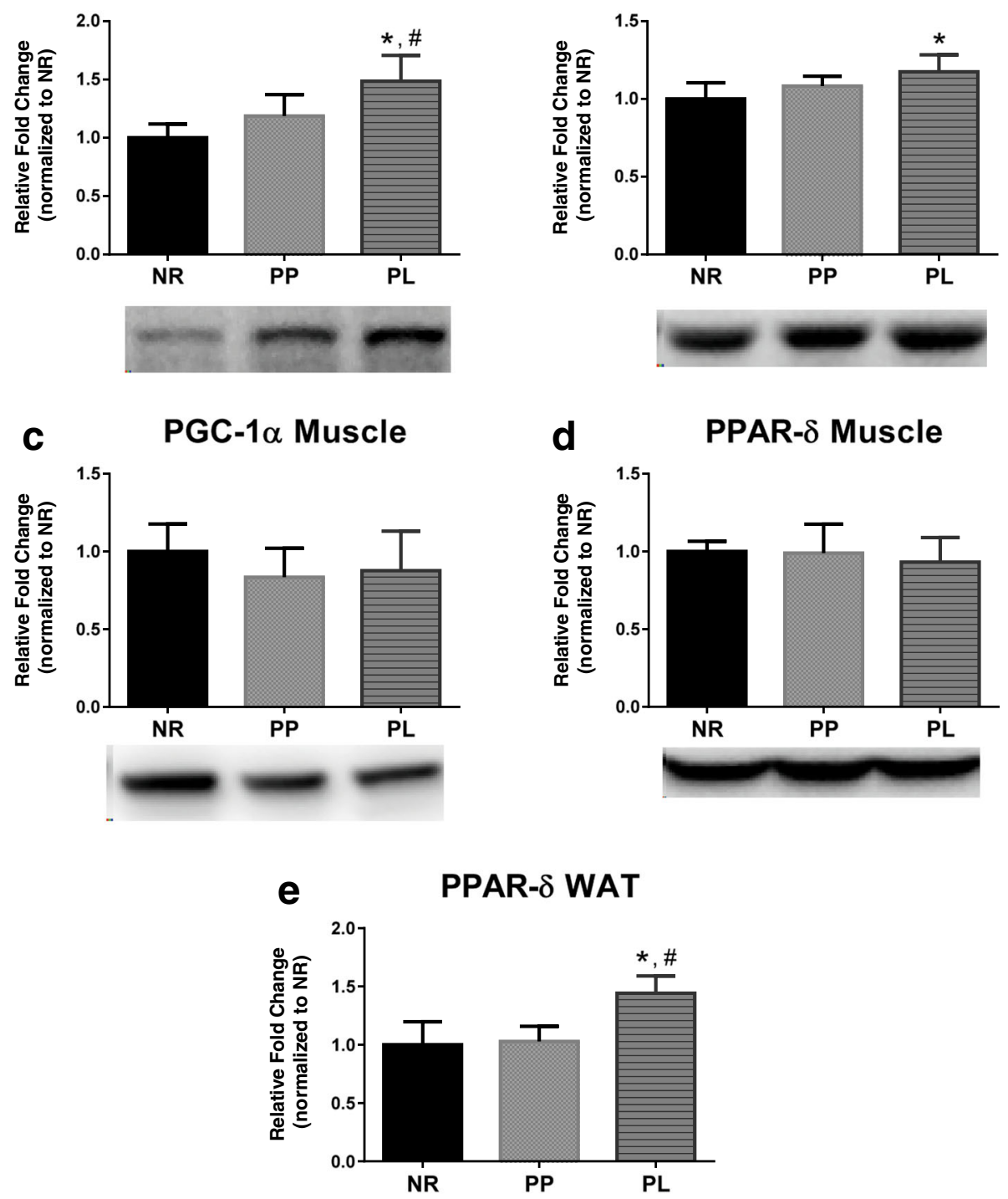

Fig. 4 Markers of metabolism in liver, skeletal muscle, and white adipose tissue (WAT) of age-matched rats that did not reproduce (NR), and rats that did not (PP) or did (PL) suckle their young for 21 days postpartum. Data include (a) PGC-1a and (b) PPARס protein levels in liver. (c) PGC-1a

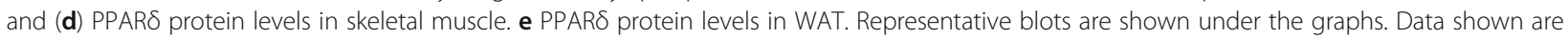
mean \pm SD. ${ }^{*}$ indicates different from NR $(p<0.05)$, and \# indicates different from PP $(p<0.05)$

body and WAT mass and lower serum glucose concentration in animals that lactated. Furthermore, our data indicate that glucose metabolism was improved, in part, due to improved liver mitochondria function.

\section{Change in body and tissue mass}

The process of milk synthesis presents a high energetic demand to the mother. In mice, resting metabolic rate during peak lactation has been recorded as being 2-fold higher than controls [33]. A large contribution to this increase in resting metabolic rate is imposed by the liver, which doubles in size, and the mammary gland that is actively synthesizing milk. Our findings show that PP rats had a higher body mass 28 days post-partum compared to NR, while no statistical differences were detected between PL and NR rats. Furthermore, our results indicate that while body mass was not different between NR and PL rats, WAT mass was lower in PL rats compared to both PP and NR rats. These 
a

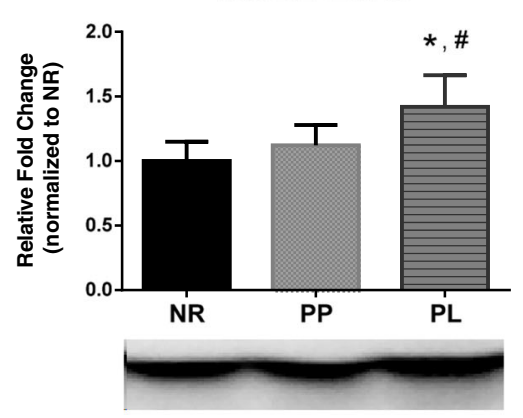

C

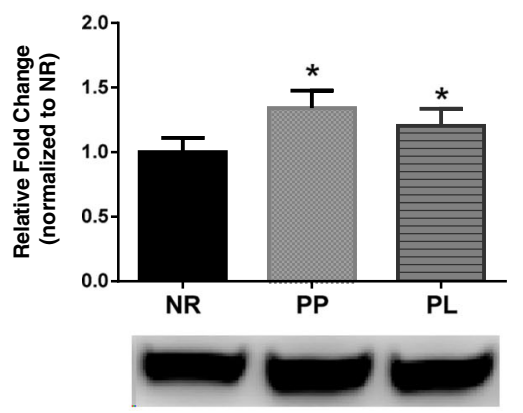

b

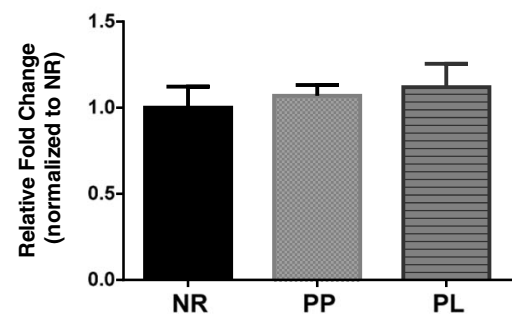

d

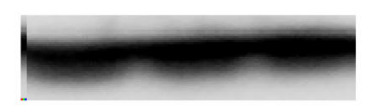

GPX Liver

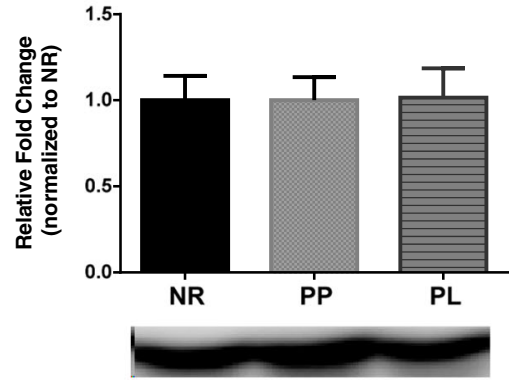

e

4-HNE Liver
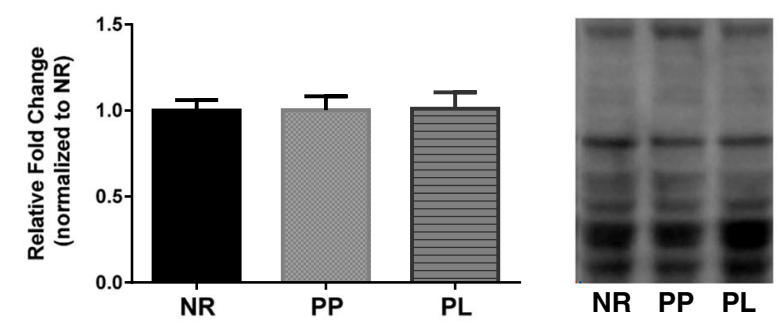

$\mathbf{f}$

Liver Protein Carbonyls
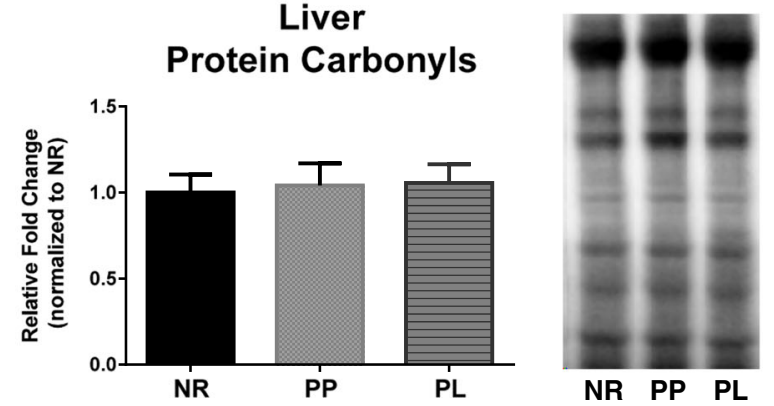

Fig. 5 Markers of oxidative stress in liver of age-matched rats that did not reproduce (NR), and rats that did not (PP) or did (PL) suckle their young for 21 days postpartum. Data include protein level of the antioxidants (a) SOD2, (b) SOD1, (c) CAT, and (d) GPX. In addition, data include markers of oxidative damage including (e) lipid peroxidation determined by 4-HNE, and (f) protein carbonyls levels determined by using oxyblot. Representative blots are shown under the graphs $(\mathbf{a}-\mathbf{d})$ or to the right of the graphs (e and $\mathbf{f})$. Data shown are mean \pm SD. * indicates different from NR $(p<0.05)$, and \# indicates different from PP $(p<0.05)$

finding agree with previous work showing lactation leads to a reduction in absolute fat mass associated with fewer visceral adipocytes [34-36]. In addition, liver is hypertrophied during gestation and lactation in order to meet the demands of higher energy utilization. Our data support this, and liver mass was still higher in PL rats 7 days after weaning, which may have masked some of the difference in body mass between the groups. These findings highlight the extent of anatomical changes that occur in response to the metabolic perturbations imposed by lactation. 


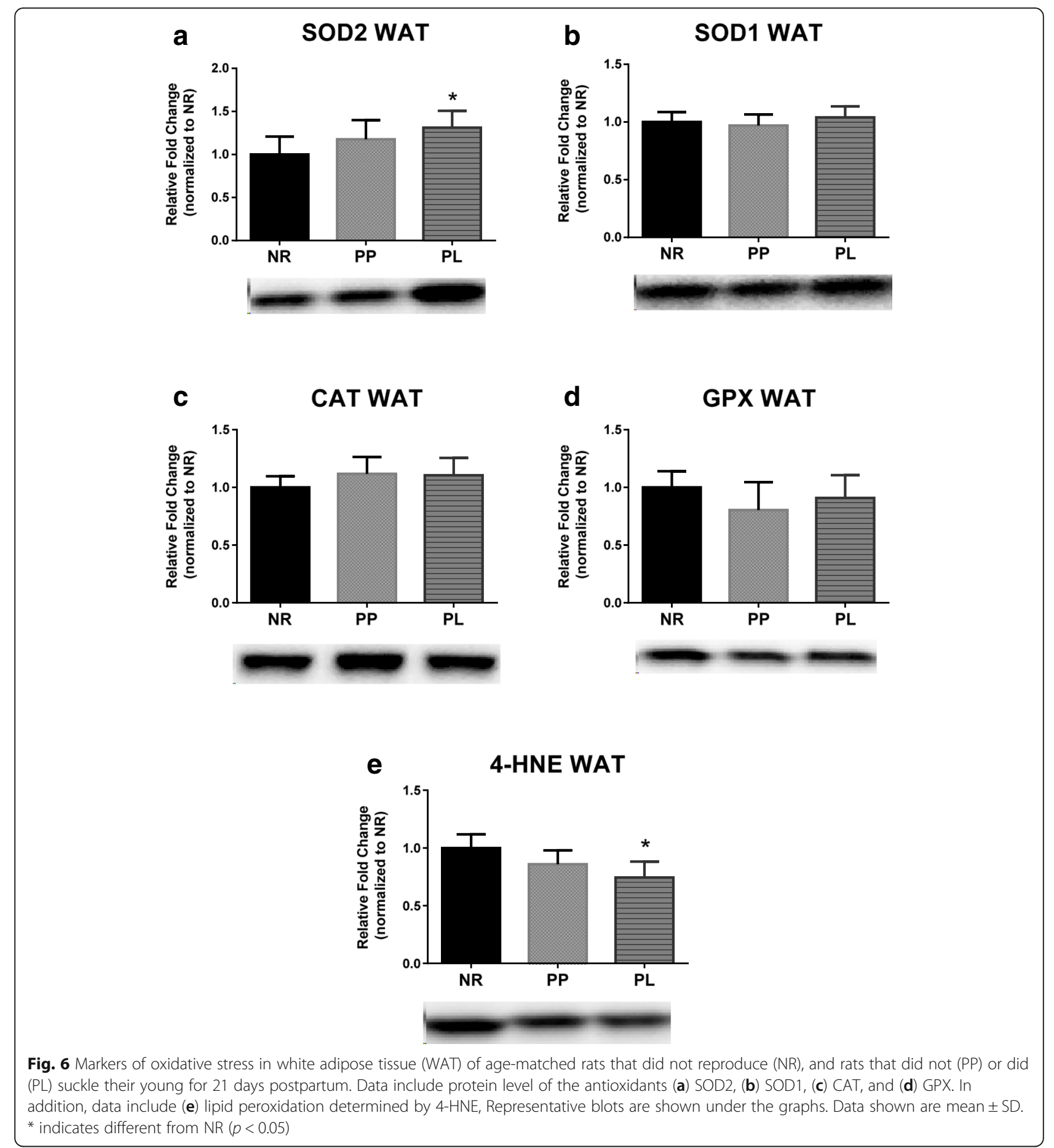

\section{Lactation and metabolism}

Systemic changes in numerous metabolic processes must occur during pregnancy for the female's body to support the growing fetus. One such change is an increase in maternal insulin production. An increase in maternal insulin production facilitates glucose transport to the fetus, but this can often result in increased insulin resistance in maternal cells. In some cases, this may manifest itself as gestational diabetes. We show that alterations of glucose metabolism due to pregnancy is sustained to some degree in females that do not participate in lactation. This idea is supported through epidemiological findings suggesting that this effect increases a woman's risk of developing type II diabetes later in life relative to women who do breastfeed [5, 37]. In contrast to pregnancy, there is a decrease in insulin secretion during lactation returning to pre-pregnancy levels 

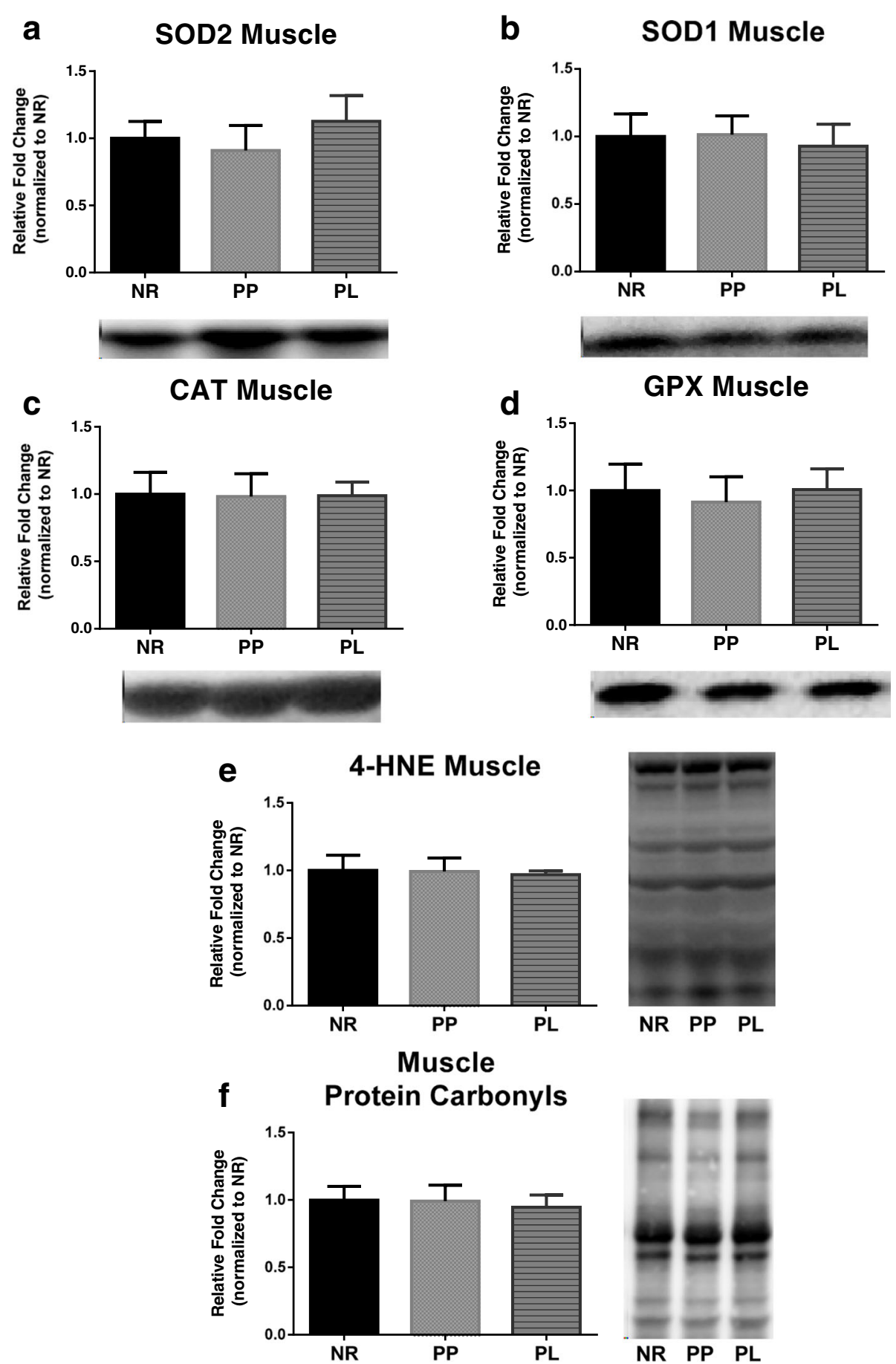

Fig. 7 Markers of oxidative stress in muscle of age-matched rats that did not reproduce (NR), and rats that did not (PP) or did (PL) suckle their young for 21 days postpartum. Data include protein level of the antioxidants (a) SOD2, (B) SOD1, (c) CAT, and (d) GPX. In addition, data include markers of oxidative damage including (e) lipid peroxidation determined by 4-HNE, and (f) protein carbonyls levels determined by using oxyblot. Representative blots are shown under the graphs (a-d) or to the right of the graphs (e and $\mathbf{f}$ ). Data shown are mean \pm SD

that is associated with a decline in $\beta$-cell proliferation and improved insulin sensitivity [13].

Importantly, our finding of higher liver protein levels of PPAR $\delta$ in PL rats may help explain how glucose metabolism may be increased due to a lactation period. PPAR $\delta$ plays a significant role in the regulation of glucose metabolism and insulin sensitivity [29] and Sanderson and collaborators demonstrated its importance in 
the liver. Specifically, knockout models of PPAR $\delta$ had lower expression of genes relating to glucose metabolism and higher plasma glucose in a fasted state compared to wild type mice [38]. These studies and our data, in which fasted serum glucose was decreased and liver PPAR $\delta$ protein expression was higher in PL rats, suggest that the resulting modulation of PPAR $\delta$ expression persists after lactation and is at least one mechanism that may confer a phenotype that protects against type II diabetes. In addition, we report that mitochondrial respiratory function was enhanced in PL rats when using complex I substrates, as indicated by a higher RCR. Considering that complex I respiration plays a large role in the utilization of glucose, via high NADH:FADH ${ }_{2}$ production, our findings provides a mechanistic outcome for the enhanced ability of cells in PL rats to metabolize glucose. Interestingly, measurements of enzymatic activity of the electron transport system show that both PP and PL rats had higher complex I activity. Our findings of increased liver complex I mitochondrial function in PL animals may be better explained by the increased protein expression of PGC- $1 \alpha$ and PPAR $\delta$. While PPAR $\delta$ knockout models have demonstrated that PPAR $\delta$ is involved in the expression of genes relating to oxidative metabolism, PGC- $1 \alpha$ is a wellknown regulator of mitochondrial biogenesis and coactivator of genes involved in oxidative phosphorylation [28]. Thus, increased expression of these proteins likely results in the improved mitochondrial function observed. PL rats also expressed lower liver complex II activity compared to both PP and NR, and lower respiration when using complex II substrates. This down regulation of complex II respiration further limits use of $\mathrm{FADH}_{2}$ to fuel OXPHOS, and as a result, likely down regulates fat metabolism via beta oxidation, an effect that further supports the use of glucose as the primary fuel to support ATP production in PL rats. During pregnancy, the mother's body experiences increasing visceral adiposity [12]. During lactation however, a shift in lipoprotein lipase and triacylglyceride levels facilitate the mobilization of fats to the mammary gland to be used for milk synthesis [14]. In contrast to PPAR $\delta$ 's role in liver, PPAR $\delta$ in WAT is involved in catabolic effects through oxidation of fatty acids [30]. Our data show that PPAR $\delta$ protein expression is higher in WAT of PL rats compared to NR and PP rats. Our findings of increased PPAR $\delta$ protein expression in two functionally different tissues also demonstrate that a systemic signaling event is a likely candidate for driving these changes.

\section{Oxidative stress in liver and WAT}

The production of reactive oxygen species (ROS) is a naturally occurring phenomenon that can manifest itself through processes of energy production and metabolism; however an imbalance between ROS production and a counteracting antioxidant system can result in oxidative damage [39]. Oxidative stress has been implicated as a driving force in the development of diabetes and other health disparities $[40,41]$ and several investigators have theorized that oxidative stress is a physiological cost of reproduction that has the potential to impact a female's future reproductive performance and longevity [42, 43]. Yet, the results of studies testing this hypothesis have provided equivocal results [18]. Our data show no differences in markers of liver lipid peroxidation and protein oxidation between the groups described here that were collected 1 week after lactation has ended, suggesting that there was no persistent oxidative cost of reproduction. With no difference in oxidant emission in liver, our finding of higher liver SOD2 in PL rats may be better explained by increased PGC- $1 \alpha$, as SOD2 is a downstream target of PGC-1 $\alpha$ [44] than as a mechanism of preventing oxidative damage. Systemic effects of prior lactation are further demonstrated by our finding that SOD2 protein levels were also higher in the WAT of PL rats. However, oxidative damage was lower in WAT of PL as shown by decreased 4-HNE. Reduced oxidative damage in WAT following lactation may serve as a beneficial adaptation, as oxidative stress in WAT has been proposed to disrupt adipokine secretion by WAT, which may play a role in the development of metabolic syndrome [41].

\section{Conclusions}

We report that rats who experienced lactation had reduced body mass, reduced WAT mass, and exhibited changes in mitochondrial function and select markers of metabolism and oxidative stress after lactation had ended and mammary tissue had regressed. Collectively these data advance our understanding of the physiological and metabolic changes that occur in animals that give birth and either suckle their pups or do not suckle their pups. In closing, these animal findings may aid future research efforts in identifying potential physiological mechanisms that mediate the persisting maternal metabolic health benefits accrued due to lactation in human populations. This topic remains an important clinical issue, as identifying these targets can be used to benefit mothers who are unable to breastfeed their young.

\footnotetext{
Abbreviations

4-HNE: 4-hydroxynoneal; BSA: Bovine serum albumin; CAT: Catalase; GPX: Glutathione peroxidase; NEFA: Non-esterified fatty acids; NR: Non-reproductive rats; PBS: Phosphate-buffered saline; PGC-1a: Peroxisome proliferator activated receptor gamma coactivator 1 alpha; PGC-1ß: Peroxisome proliferator activated receptor gamma coactivator 1 beta; $\mathrm{PL}$ : Rats that allowed to mate, became pregnant, and suckled their young until weaning at 21 days; PP: Rats that allowed to mate and became pregnant, but were not allowed to suckle their pups; PPARa: Peroxisome proliferator activated receptor alpha; PPARס: Peroxisome proliferator activated receptor delta; RCR: Respiratory control ratio; SOD1: Superoxide dismutase 1; SOD2: Superoxide dismutase 2; WAT: White adipose tissue
} 


\section{Acknowledgements}

Not applicable.

\section{Funding}

This research was funded by US National Institutes of Health grant R03 HD083654-01

\section{Availability of data and materials}

Data are not publicly shared; please contact the authors for data requests.

\section{Authors' contributions}

HWH, YZ, WRH, ANK contributed to the design and conduct of the study data collection and analysis, data interpretation and manuscript writing. All authors read and approved the final manuscript.

\section{Ethics approval}

All experimental procedures were approved by Auburn University's Institutional Animal Care and Use Committee (PRN 2014-2591).

\section{Consent for publication}

Not applicable.

\section{Competing interests}

The authors declare that they have no competing interests.

\section{Publisher's Note}

Springer Nature remains neutral with regard to jurisdictional claims in published maps and institutional affiliations.

\section{Author details}

'School of Kinesiology, Auburn University, 301 Wire Road, Auburn, Alabama 36849, USA. ${ }^{2}$ Department of Biological Sciences, Auburn University, Auburn, AL, USA.

Received: 30 September 2017 Accepted: 25 December 2017 Published online: 09 January 2018

\section{References}

1. Colberg SR, Sigal RJ, Fernhall B, Regensteiner JG, Blissmer BJ, Rubin RR, Chasan-Taber L, Albright AL, Braun B. American College of Sports M, American diabetes a: exercise and type 2 diabetes: the American College of Sports Medicine and the American Diabetes Association: joint position statement. Diabetes Care. 2010;33:e147-67.

2. Klempel MC, Kroeger CM, Bhutani S, Trepanowski JF, Varady KA. Intermittent fasting combined with calorie restriction is effective for weight loss and cardio-protection in obese women. Nutr J. 2012;11:98.

3. Thomas JA 2nd, Antonelli JA, Lloyd JC, Masko EM, Poulton SH, Phillips TE, Pollak M, Freedland SJ. Effect of intermittent fasting on prostate cancer tumor growth in a mouse model. Prostate Cancer Prostatic Dis. 2010;13:350-5.

4. Heilbronn LK, Ravussin E. Calorie restriction and aging: review of the literature and implications for studies in humans. Am J Clin Nutr. 2003;78:361-9.

5. Natland ST, Lund Nilsen T, Midthjell K, Frost A, Forsmo S. Lactation and cardiovascular risk factors in mothers in a population-based study: the HUNT-study. Int Breastfeed J. 2012;7:8.

6. Ram KT, Bobby P, Hailpern SM, Lo JC, Schocken M, Skurnick J, Santoro N. Duration of lactation is associated with lower prevalence of the metabolic syndrome in midlife-SWAN, the study of women's health across the nation. Am J Obstet Gynecol. 2008;198:268. e261-e266

7. Stuebe AM, Kleinman K, Gillman MW, Rifas-Shiman SL, Gunderson EP, RichEdwards J. Duration of lactation and maternal metabolism at 3 years postpartum. J Women's Health. 2010;19:941-50.

8. Stuebe AM, Michels KB, Willett WC, Manson JE, Rexrode K, Rich-Edwards JW. Duration of lactation and incidence of myocardial infarction in middle to late adulthood. Am J Obstet Gynecol. 2009;200:138. e131-138. e138

9. Stuebe AM, Rich-Edwards JW. The reset hypothesis: lactation and maternal metabolism. Am J Perinatol. 2009:26:81.

10. Ramos P, Herrera E. Effect of prolonged glucose infusion on insulin sensitivity in the conscious normal rat. Horm Metab Res. 1995;27:197-200.

11. Martin A, Zorzano A, Caruncho I, Herrera E. Glucose tolerance tests and "in vivo" response to intravenous insulin in the unanaesthesized late pregnant rat and their consequences to the fetus. Diabete Metab. 1986;12:302-7.
12. Einstein FH, Fishman S, Muzumdar RH, Yang XM, Atzmon G, Barzilai N. Accretion of visceral fat and hepatic insulin resistance in pregnant rats. Am J Physiol Endocrinol Metab. 2008;294:E451-5.

13. Jones RG, llic V, Williamson DH. Physiological significance of altered insulin metabolism in the conscious rat during lactation. Biochem J. 1984;220:455-60.

14. Hamosh M, Clary TR, Chernick SS, Scow RO. Lipoprotein lipase activity of adipose and mammary tissue and plasma triglyceride in pregnant and lactating rats. Biochim Biophys Acta. 1970;210:473-82.

15. Rolfe DF, Brown GC. Cellular energy utilization and molecular origin of standard metabolic rate in mammals. Physiol Rev. 1997;77:731-58.

16. Gutgesell A, Ringseis R, Schmidt E, Brandsch C, Stangl Gl, Eder K. Downregulation of peroxisome proliferator-activated receptor $a$ and its coactivators in liver and skeletal muscle mediates the metabolic adaptations during lactation in mice. J Mol Endocrinol. 2009;43:241-50.

17. Pichaud N, Garratt M, Ballard JW, Brooks RC. Physiological adaptations to reproduction. II. Mitochondrial adjustments in livers of lactating mice. J Exp Biol. 2013;216:2889-95.

18. Speakman JR, Garratt M. Oxidative stress as a cost of reproduction: beyond the simplistic trade-off model. BioEssays. 2014:36:93-106.

19. Hyatt HW, Kephart WC, Holland AM, Mumford P, Mobley CB, Lowery RP, Roberts MD, Wilson JM, Kavazis AN. A Ketogenic diet in rodents elicits improved mitochondrial adaptations in response to resistance exercise training compared to an Isocaloric western diet. Front Physiol. 2016;7:533.

20. Mowry AV, Kavazis AN, Sirman AE, Potts WK, Hood WR. Reproduction does not adversely affect liver mitochondrial respiratory function but results in lipid peroxidation and increased antioxidants in house mice. PLoS One. 2016;11:e0160883.

21. Messer Jl, Jackman MR, Willis WT. Pyruvate and citric acid cycle carbon requirements in isolated skeletal muscle mitochondria. Am J Physiol Cell Physiol. 2004;286:C565-72.

22. Wanders RJ, Groen AK, Van Roermund CW, Tager JM. Factors determining the relative contribution of the adenine-nucleotide translocator and the ADP-regenerating system to the control of oxidative phosphorylation in isolated rat-liver mitochondria. Eur J Biochem. 1984;142:417-24.

23. Estabrook RW. [7] mitochondrial respiratory control and the polarographic measurement of ADP: O ratios. Methods Enzymol. 1967:10:41-7.

24. Kavazis AN, Talbert EE, Smuder AJ, Hudson MB, Nelson WB, Powers SK. Mechanical ventilation induces diaphragmatic mitochondrial dysfunction and increased oxidant production. Free Radic Biol Med. 2009;46:842-50.

25. Trounce IA, Kim YL, Jun AS, Wallace DC. Assessment of mitochondrial oxidative phosphorylation in patient muscle biopsies, lymphoblasts, and transmitochondrial cell lines. Methods Enzymol. 1996;264:484-509.

26. Bradford MM. A rapid and sensitive method for the quantitation of microgram quantities of protein utilizing the principle of protein-dye binding. Anal Biochem. 1976;72:248-54.

27. Barja G. Mitochondrial oxygen radical generation and leak: sites of production in states 4 and 3, organ specificity, and relation to aging and longevity. J Bioenerg Biomembr. 1999;31:347-66

28. Wu Z, Puigserver $P$, Andersson U, Zhang C, Adelmant G, Mootha V, Troy A Cinti S, Lowell B, Scarpulla RC, Spiegelman BM. Mechanisms controlling mitochondrial biogenesis and respiration through the thermogenic coactivator PGC-1. Cell. 1999;98:115-24.

29. Lee $\mathrm{CH}$, Olson P, Hevener A, Mehl I, Chong LW, Olefsky JM, Gonzalez FJ, Ham J Kang $\mathrm{H}$, Peters JM, Evans RM. PPARdelta regulates glucose metabolism and insulin sensitivity. Proc Natl Acad Sci U S A. 2006:103:3444-9.

30. Roberts LD, Murray AJ, Menassa D, Ashmore T, Nicholls AW, Griffin JL. The contrasting roles of PPAR $\delta$ and PPARg in regulating the metabolic switch between oxidation and storage of fats in white adipose tissue. Genome Biol. 2011;12:R75.

31. Barnosky AR, Hoddy KK, Unterman TG, Varady KA. Intermittent fasting vs daily calorie restriction for type 2 diabetes prevention: a review of human findings. Transl Res. 2014;164:302-11.

32. Ross R, Dagnone D, Jones PJ, Smith H, Paddags A, Hudson R, Janssen I. Reduction in obesity and related comorbid conditions after diet-induced weight loss or exercise-induced weight loss in men. A randomized, controlled trial. Ann Intern Med. 2000;133:92-103.

33. Speakman J, McQueenie J. Limits to sustained metabolic rate: the link between food intake, basal metabolic rate, and morphology in reproducing mice, Mus Musculus. Physiol Zool. 1996:69:746-69.

34. Moore BJ, Brasel JA. One cycle of reproduction consisting of pregnancy, lactation or no lactation, and recovery: effects on fat pad cellularity in ad libitum-fed and food-restricted rats. J Nutr. 1984;114:1560-5. 
35. Moore BJ, Brasel JA. One cycle of reproduction consisting of pregnancy, lactation or no lactation, and recovery: effects on carcass composition in ad libitum-fed and food-restricted rats. J Nutr. 1984;114:1548-59.

36. Moore BJ, Olsen JL, Marks F, Brasel JA. The effects of high fat feeding during one cycle of reproduction consisting of pregnancy, lactation and recovery on body composition and fat pad cellularity in the rat. J Nutr. 1984;114:1566-73.

37. Stuebe AM, Rich-Edwards JW, Willett WC, Manson JE, Michels KB. Duration of lactation and incidence of type 2 diabetes. JAMA. 2005;294:2601-10.

38. Sanderson LM, Boekschoten MV, Desvergne B, Muller M, Kersten S. Transcriptional profiling reveals divergent roles of PPARalpha and PPARbeta/ delta in regulation of gene expression in mouse liver. Physiol Genomics. 2010;41:42-52

39. Powers SK, Ji L, Leeuwenburgh C. Exercise training-induced alterations in skeletal muscle antioxidant capacity: a brief review. Med Sci Sports Exerc. 1999;31:987-97.

40. Baynes JW. Role of oxidative stress in development of complications in diabetes. Diabetes. 1991;40:405-12.

41. Furukawa S, Fujita T, Shimabukuro M, Iwaki M, Yamada Y, Nakajima Y, Nakayama O, Makishima M, Matsuda M, Shimomura I. Increased oxidative stress in obesity and its impact on metabolic syndrome. J Clin Invest. 2004; 114:1752-61.

42. Dowling DK, Simmons LW. Reactive oxygen species as universal constraints in life-history evolution. Proc Biol Sci. 2009;276:1737-45.

43. Monaghan P, Metcalfe NB, Torres R. Oxidative stress as a mediator of life history trade-offs: mechanisms, measurements and interpretation. Ecol Lett. 2009;12:75-92

44. Lu Z, Xu X, Hu X, Fassett J, Zhu G, Tao Y, Li J, Huang Y, Zhang P, Zhao B, Chen Y. PGC-1 alpha regulates expression of myocardial mitochondrial antioxidants and myocardial oxidative stress after chronic systolic overload. Antioxid Redox Signal. 2010;13:1011-22.

\section{Submit your next manuscript to BioMed Central and we will help you at every step:}

- We accept pre-submission inquiries

- Our selector tool helps you to find the most relevant journal

- We provide round the clock customer support

- Convenient online submission

- Thorough peer review

- Inclusion in PubMed and all major indexing services

- Maximum visibility for your research

Submit your manuscript at www.biomedcentral.com/submit 